\title{
Entanglement, symmetry breaking and collapse: correspondences between quantum and self-organizing dynamics
}

\author{
Francis Heylighen \\ Center Leo Apostel, \\ Vrije Universiteit Brussel, Pleinlaan 2, \\ 1050 Brussel, Belgium \\ fheyligh@vub.ac.be
}

\begin{abstract}
Quantum phenomena are notoriously difficult to grasp. The present paper first reviews the most important quantum concepts in a non-technical matter: superposition, uncertainty, collapse of the wave function, entanglement and non-locality. It then tries to clarify these concepts by examining their analogues in complex, self-organizing systems. These include bifurcations, attractors, emergent constraints, order parameters and non-local correlations. They are illustrated with concrete examples that include Rayleigh-Bénard convection, social self-organization and Gestalt perception of ambiguous figures. In both cases, quantum and self-organizing, the core process appears to be a symmetry breaking that irreversibly and unpredictably "collapses" an ambiguous state into one of a number of initially equivalent "eigenstates" or "attractors". Some speculations are proposed about the non-linear amplification of quantum fluctuations of the vacuum being ultimately responsible for such symmetry breaking.
\end{abstract}

Keywords: quantum processes, self-organization, entanglement, collapse of the wave function, symmetry breaking, bifurcation

\section{Introduction}

Quantum mechanics is a theory notorious for the fact that the phenomena it describes are highly counterintuitive. What makes it particularly difficult for us to imagine what happens is that these phenomena belong to a microworld that we can never hope to perceive with our senses. Moreover, their description uses a highly abstract mathematical formalism (operators in Hilbert spaces) that has no clear counterpart in other, more intuitive theories of the physical world. Nevertheless, we may be able to understand these quantum phenomena better if we could find analogues of them in the 
macroworld in which we live. One recently developed example of this approach is quantum cognition (Aerts, 2009; Bruza, Wang, \& Busemeyer, 2015): establishing a correspondence between cognitive processes in our brain and quantum mechanisms. But the brain is an extremely complex system of which we as yet do not understand too much. Therefore, it is not yet clear in how far the apparent similarities between cognitive and quantum structures will help us to elucidate either the one or the other.

The present article proposes to examine the analogies between quantum systems and systems that are still complex, but not as complex as the brain, and to which we have a more direct access, both empirically and theoretically. I am referring to what is known as complex systems, complex adaptive systems, or self-organizing systems (Ball, 2012; Heylighen, 2009). These are systems that consist of many interacting components, typically modeled as "agents", that are distributed in space. Agents can be molecules, people, insects, or neurons. Local interactions between agents commonly give rise to globally coordinated behavior, as exemplified by the movement of birds in a swarm, ants in a colony, or fish in a shoal. Such emergence of order or coherence is called self-organization (Ashby, 1962; Haken, 1977; Heylighen, 2001). This is a non-linear process that tends to amplify tiny fluctuations into macroscopic differences. As a result, such complex processes are typically unpredictable and difficult to control - a property they share with quantum systems. But, as I will elaborate in the remainder of this paper, the similarities go much deeper.

I will first briefly review the most characteristic and poorly understood concepts of quantum mechanics, such as non-locality, entanglement and collapse of the wave function, in a non-technical manner. I will then review related concepts in complex systems theory. Using examples, I will try to establish a correspondence between each of the core quantum concepts and its counterpart in complexity. Finally, I will make some suggestions about how this correspondence may help us to understand the counterintuitive aspects of quantum theory.

\section{Core quantum concepts}

Probably the most fundamental difference between a quantum theory and a classical theory of some physical system is that the quantum model obeys the superposition principle (Dirac, 1981; Heylighen, 1990). This means that for any two states $a$ and $b$ of the system that can be distinguished there exists some third state $a+b$, called a superposition of $a$ and $b$. This superposition state has properties that somehow combine the properties of $a$ with those of $b$. In other words, this state is not in between $a$ and $b$, it is somehow both $a$ and $b$. For example, suppose that the possible states of your system are $\mid$ black $>$ and $\mid$ white $>$. Classically, you might expect that there exists a separate, "inbetween" state, such as $\mid$ grey $>$. But in a quantum theory, there would always be a superposition state $\mid$ black $>+\mid$ white $>$, which would sometimes appear as black and sometimes as white, but never as grey.

The name "superposition" comes from the representation of quantum states as wave functions. Here, quantum systems are viewed as waves that spread out over space, with different intensities (ultimately determining the probabilities of finding the system) in different positions. Superposing two waves means that you add the intensities for each of the positions to get the total 
intensity of the combined wave for that position. If one wave would have a non-zero intensity only in region $a$ (meaning that the probability to find it outside that region is nil) and the other in region $b$, then the superposed wave would be present in both $a$ and $b$, but nowhere else-no matter how far apart these two regions are. This means that the particle is in a sense distributed across these two regions, somehow being in both at the same time.

This operation of addition of wave functions is straightforwardly generalized to the addition of vectors, which form an equivalent mathematical representation of quantum states. The superposition principle can then be expressed by the fact that the set of all states of a quantum system defines a particular type of vector space, called "Hilbert space". This means that any linear combination of states (vectors) defines another possible state (vector) in the state space of the system. Still, we do not need the Hilbert space formalism to understand the core concept of superposition: for any two distinct states of the system there exists at least one other state that is neither the one, nor the other, but that somehow has the properties of both. This ambivalence creates an intrinsic indeterminacy at the heart of any quantum theory.

But how can something be both black and white at the same time? Ultimately, the ambiguity is resolved by the process of observation. When an experiment is performed to ascertain what the color of the system is, the result can only be one of the colors. That means that the superposition state black + white will give either the result "white" or the result "black". However, we cannot predict beforehand which result it will be. This brings us to another core feature of the quantum theory: uncertainty or indeterminacy. A state such that the property to be observed has a determinate value is called an "eigenstate" of the observable property. If the system is not in a superposition state, but in such an eigenstate (e.g. it is in the state black), then the probability of the result black will be 1. However, since there are many more superpositions than eigenstates, in the most general case, a quantum system will be in a superposition state for the particular feature we want to determine. Therefore, we cannot say which result the observation will produce. Given the specific form of the vector or wave function, we can merely calculate the probabilities of the different outcomes.

On the other hand, if an observation is repeated, the result will not change: black remains black. This is explained in the quantum formalism by the projection postulate. The vector black + white is orthogonal neither to the black vector nor to the white vector. When the observation is made, it is somehow projected onto one of its orthogonal component vectors, the eigenstates white or black. Once projected onto, say, black, the state has effectively become identical to the one it was projected upon (because a state only depends on the direction of the vector, not its length). So now it is $100 \%$ black, without ambiguity.

This process is traditionally called the collapse of the wave function. Suppose that the wave function (which is a representation of a state equivalent to the vector representation) is spread out over two separate regions, one corresponding to black, one to white. Performing the operation of observation forces the wave to choose one of the two possible outcomes, white or black. That means that after the observation it is fully concentrated in one region, say black, while it has vanished from the other region. The wave has "collapsed" from a wider region, covering both black and white, to a smaller one, covering only black. 
This "projection" or "collapse" is presented in quantum mechanics as an abstract mathematical operation, not as a concrete physical process. Therefore, the collapse is assumed to happen instantaneously, even though in practice the experiment needed to make an observation will of course need some time to take place. Yet, the quantum theory says nothing about what is supposed to happen during that time; it only describes the state before (superposition state) and after (projected state) that operation. Thus, the collapse of the wave function is another highly mysterious and counterintuitive quantum property: it is as if the wave "jumps" instantaneously from one region to another one without passing through any intermediate regions. This will lead us to another bizarre quantum feature: non-locality.

Entanglement is a quantum property that follows straight from superposition (Horodecki, Horodecki, Horodecki, \& Horodecki, 2009). Assume that you have two quantum systems (typically particles), $x$ and $y$. Each of them can be in a particular state, e.g. $\mid x$-black $>$, and $\mid y$-white $>$. The state of the compound system is then $\mid x$-black $>\mid y$-white $>$. But this compound state, describing the system consisting of both $x$ and $y$, also obeys the superposition principle. Therefore, the compound system can be in the superposition state:

$$
\mid x \text {-black }>\mid y \text {-white }>+\mid x \text {-white }>\mid y \text {-black }>\text {. }
$$

This means that if you observe $x$ and find the result black, the compound state will have collapsed to the first part of the sum: $\mid x$-black $>y$-white $>$. Therefore, any further observation of $y$ will necessarily produce the result white. However, if the first observation of $x$ had found white as result, the collapse of the wave function would have forced the observation of $y$ to produce black. In this case, we say that the components $x$ and $y$ are entangled, because the observation of the one cannot be separated from the observation of the other. Before the observation, both $x$ and $y$ could have produced the result white or black. But once a result for one of the components is determined, the one for the other is determined as well. The results mutually determine each other, and cannot be disentangled.

A final quantum concept we need to introduce is non-locality (Wiseman, 2006). Suppose that components $x$ and $y$ are separated by a distance in space. For example, $x$ might reside in Brussels, and $y$ in Tokyo. Assume that both systems are entangled, i.e. their compound state is a superposition of two states each characterized by different individual values (black, white) for each of the components. An observation of $x$ in Brussels will determine the value of its property (say, black). But because the collapse of the wave function is instantaneous, this means that at the same time the value of $y$ in Tokyo will become determined (in this case to white). The outcomes of the two experiments are correlated: whenever the one results in black, the other one will necessarily result in white. The distance between the two components of the system is completely irrelevant to this process. Therefore, it does not depend on their locality: the collapse is non-local. It is as if quantum observation processes ignore distance or space. The state of the compound system is simply smeared out or distributed across different locations, no matter how far or how near the locations are from each other.

At first sight, non-locality contradicts the basic principle of relativity theory, which says that signals cannot travel faster than the speed of light. The instantaneity of the collapse implies that the 
state of $y$ is reduced to black the very moment the one of $x$ is determined to be white. This leaves no time for a signal to travel from Brussels to Tokyo that would "tell" the particle $y$ that it should collapse to its black state. Yet, numerous experiments with entangled systems that are observed simultaneously have confirmed that such a collapsed outcome indeed results before any signal traveling with a speed not faster than light could have reached the second component (Aspect, 2007). Does quantum theory then prove relativity theory to be wrong? Not at all, because whatever is transmitted between the two components is not a "signal", in the sense that it cannot be used to transfer information from the one location to the other. This has been proven mathematically using different versions of the quantum formalism (Eberhard \& Ross, 1989; Ghirardi, Grassi, Rimini, \& Weber, 1988).

We can understand this result more intuitively by noting that an observer in Tokyo cannot determine whether the component on that side has collapsed because of some measurement in Brussels. When the Tokyo observer measures the property of the component, s/he will find "black" with $50 \%$ probability, no matter whether a previous measurement was or was not performed in Brussels. Finding "black" in Tokyo may mean that the Brussels observer found "white" and thus collapsed the Tokyo component to its "black" state just before the Tokyo experiment. But it may equally mean that the Tokyo observer collapsed the system to its "black" state in Tokyo, before any Brussels experiment took place. The local experiment in Tokyo or Brussels cannot ascertain whether an experiment in the other location has or has not taken place. Only after we bring together the results of several such experiments in both locations will we be able to infer from the correlations between their results that the components were entangled. And that bringing together still requires the transmission of information with a velocity not higher than the speed of light, meaning that relativity theory is not contradicted...

In summary, what we need to remember from quantum theory is that systems can be in some indeterminate superposition state, which is neither one thing or its opposite, nor something in between, but in a sense both things at the same time. Moreover, the result from observing such a superposition state is fundamentally uncertain, and can only be predicted statistically. After the observation, the superposition state "collapses" to the eigenstate that corresponds to the value measured, and remains in that state when the observation is repeated. This collapse is instantaneous, even when the components of the system observed are arbitrarily far apart. The components of a system in a superposition state are said to be entangled. The collapse means that entangled components instantaneously influence each other's state, independently of the distance between them, a feature called non-locality.

\section{Core concepts of complex dynamics}

The theory of complex systems is as yet much less developed than quantum mechanics. Thus, its concepts are more diverse, less clearly defined and less integrated into a coherent theory. There is in particular no overall formalism, like the one defined for quantum mechanics by the axioms formulated by von Neumann. Therefore, I will here merely present a selection of what I consider to 
be some of its more fundamental ideas, with a focus on those that seem most similar to quantum concepts.

Complex systems consist of many components that undergo many, non-trivial interactions, so that they are impossible to separate or reduce to independent aspects or properties (Heylighen, 2009; Heylighen, Cilliers, \& Gershenson, 2007). Therefore, it is in general not possible for an observer to collect complete information about the state of the system. Statistical mechanics has developed dependable methods to model systems, such as gases, that have many components but that lack such intricate, non-linear interactions and dependencies. Since it is impossible to measure the position and velocity of each individual gas molecule, we cannot determine the full "microstate" of the system. However, since a gas is statistically homogeneous, a large array of such molecules can be accurately described by macroscopic properties such as volume, temperature and pressure, together determining the "macrostate" of the system. A macrostate is simply a class of microstates that are considered equivalent, in the sense that e.g. the average velocity of the molecules in each of the microstates composing a macrostate is the same. Thus, a macrostate may not provide full information about the details of the system, but it is not ambiguous, in the sense that it can normally be clearly established in which of two macrostates the system resides. Macrostates do not overlap, and a particular microstate belongs to either one or another of the distinguishable macrostate. Therefore, there is no equivalent of superpositions for macrostates.

This assumption of independence of observable states can no longer be made for complex systems. This is because we not only cannot observe the microscopic properties of each component; we also cannot determine their macroscopic interactions. This is a general feature of non-linear systems that is technically known as "sensitive dependence on initial conditions", and more informally as "the butterfly effect" (Hilborn, 2004). Non-linearity means that the effects of interactions are not proportional to their causes. This may result in an amplification of effects such that microscopic differences too small to be observed eventually produce huge, macroscopic differences. Thus, we may have two states of the system that are macroscopically indistinguishable initially (they belong to the same "macrostate"), but that still produce macroscopically distinct result after some observation is performed. For example, a state with or without the movement of a butterfly wing may result in either a hurricane or no hurricane.

Another example would be the prediction of elections through polls: when the differences in percentage of vote intentions are small enough, it becomes impossible to predict which of the candidates will get a majority. This is not just because the polls do not collect enough data: the collection and publishing of the data itself may affect the result, which is why in most countries it is forbidden to publish poll results just before the election. Even without this "observer effect", the nonlinear interactions between voters influencing each other's preferences so that they change the intentions they expressed during the poll can magnify tiny fluctuations into swings large enough to carry the majority.

In such cases, we can say that the properties of the macrostate (as determined by polls) are indeterminate: a further observation (e.g. an actual election) can produce one of several results (e.g. either candidate $a$ or candidate $b$ is elected). Initially, the system is in the equivalent of a superposition state: $a+b$. After the observation, the state has collapsed to one of the possible 


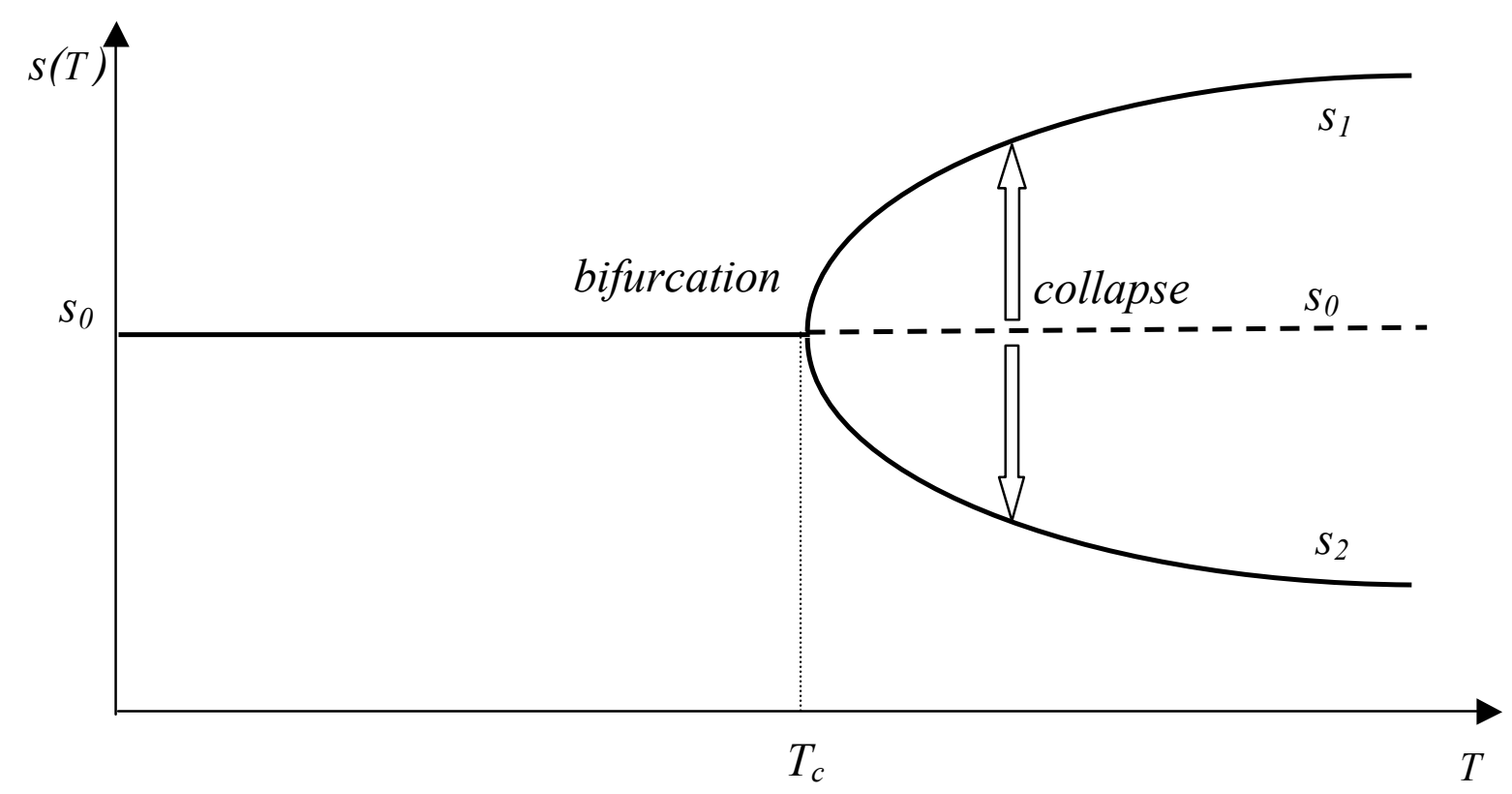

Fig. 1: the equilibrium state $s$ of a system as a function of an order parameter $T$. As $T$ reaches the critical value $T_{c}$, the number of possible states bifurcates into $s_{1}$ and $s_{2}$, while the original state $s_{0}$ becomes unstable. This instability implies that beyond the bifurcation point, $s_{0}$ must "collapse" into either $s_{1}$ or $s_{2}$, thus breaking the symmetry between the options.

outcomes: $a$ or $b$. Before the observation, we were uncertain about the result, and able at most to estimate a probability for each of the possible outcomes. But once the result was produced, it is definitive or irreversible.

This intuitive illustration can be made more precise using the mathematical notion of a bifurcation (Nicolis \& Prigogine, 1977). Assume that you have a dynamic system governed by a differential or difference equation that describes the state $s$ as a function of time $t: s(t)$. The equilibrium solutions of the equation are the ones for which $s$ is constant: $s(t)=s_{0}$. Assume that these solutions depend on a parameter $T$ (commonly called "order parameter") that characterizes the dynamics. In typical non-linear systems, like the Bénard convection that we will discuss further, there is a single solution for low values of $T$ (see Fig. 1). However, as the value of $T$ increases, you reach a point where the equation has two stable solutions: $s_{1}$ and $s_{2}$. The point in parameter space where the number of solutions changes is called the bifurcation point. The equilibrium solution $s_{0}$ still exists beyond that point but it is no longer stable: the tiniest perturbation or fluctuation away from the state $s_{0}$ makes the system shift immediately to one of the stable solutions, $s_{1}$ or $s_{2}$, where it then remains.

This shift from the unstable equilibrium $s_{0}$ to one of the stable states $s_{1}$ or $s_{2}$ can be seen as a "collapse" of the superposition state $s_{1}+s_{2}$, "projecting" it to one of its component states $s_{1}$ or $s_{2}$. The increase of the order parameter $T$ beyond the bifurcation point plays the role of an observation, which forces the system to make a choice between one of the "eigenstates" of this observation. Assuming that $s_{1}$ and $s_{2}$ are equivalent solutions, this choice for the one over the other can be seen as a symmetry breaking (Castellani, 2003). It is as if the system when arriving at the bifurcation is forced to decide whether to go left $\left(s_{1}\right)$ or right $\left(s_{2}\right)$, since it cannot continue straight on its $s_{0}$ course (which would be the only way to maintain the left-right symmetry). Like in the quantum case, this process is 
both unpredictable in its result and irreversible, in the sense that once the choice for one of the states $s_{1}$ or $s_{2}$ is made, the system remains in that state for any similar "observation".

More generally, a dynamic system is characterized by a number of attractors: regions in its state space that it can enter but not leave, and that do not contain smaller such regions (Heylighen, 2001; Milnor, 2006). Stable states like $s_{1}$ and $s_{2}$ are zero-dimensional, point attractors. Limit cycles, which commonly occur in non-linear, far-from-equilibrium systems, are one-dimensional attractors. But attractors can have any number of dimensions, including fractal. An attractor $A$ is surrounded by its basin of attraction $B(A)$. This consists of all the states in the state space whose trajectory ends up in the attractor $A$. The boundaries between basins correspond to bifurcation points (such as the unstable state $s_{0}$ ): a system on such a boundary has to make a choice about which of the two adjacent basins $B\left(A_{1}\right)$ or $B\left(A_{2}\right)$, and therefore attractors $A_{1}$ or $A_{2}$, it will enter.

A system that has reached an attractor is constrained in its further evolution, because its trajectory by definition cannot leave that attractor and move to a different attractor or basin. It has lost part of its freedom. If the state space has dimension $n$, and the attractor dimension $k<n$, then the system has lost $n-k$ of its degrees of freedom. If the system consists of different components, this means that the components in general can no longer move independently of each other. For example, assume that the system consists of two components, each with $m$ degrees of freedom. Then the system as a whole has $n=2 \times m$ degrees of freedom. After reaching the attractor, it has $k<2 \times m$ degrees of freedom, implying that its components can no longer fully use their own $m$ degrees of freedom: the movement of one component through its state space will constrain the movement of the other: their movements can no longer be separated.

As an example, consider two billiard balls, each able to move on a 2-dimensional billiard table. The two-ball system thus has $2 \times 2=4$ degrees of freedom. Assume that the balls are magnetic, so that they attract each other. After some independent movements across the table, they are likely to come so close together that they can no longer resist the attractive force and end up sticking together. This produces a two-ball assembly shaped like a figure 8 . This stuck together configuration is an attractor for the system, as the balls no longer can separate and thus recover their independent movement. This shape- 8 configuration can still move as a whole on the billiard table: 2 degrees of freedom. Moreover, it can still rotate around its axis: 1 degree of freedom. However, the balls have lost the freedom to vary the distance between them. Thus, the system now has only 3 degrees of freedom. Using quantum terminology, we could say that the balls have become entangled. Whenever we observe the position of one of the balls, we can deduce that the other ball is at a fixed distance of that position. If we were uncertain about the position of the two balls, a measurement of a single ball not only reduces our uncertainty about that ball, but also about the other one.

Thus, quantum entanglement corresponds to the complex systems phenomenon of a global constraint. This constraint on the whole forces the components of the system to behave in a coordinated manner (downward causation), while defining an emergent property (in this case the angle of rotation). Such an emergent property characterizes the way the components cohere or depend on each other, while being undefined at the level of the separate components. Therefore, it cannot be reduced to the properties of these components (Bedau, 2002; Heylighen et al., 2007). 


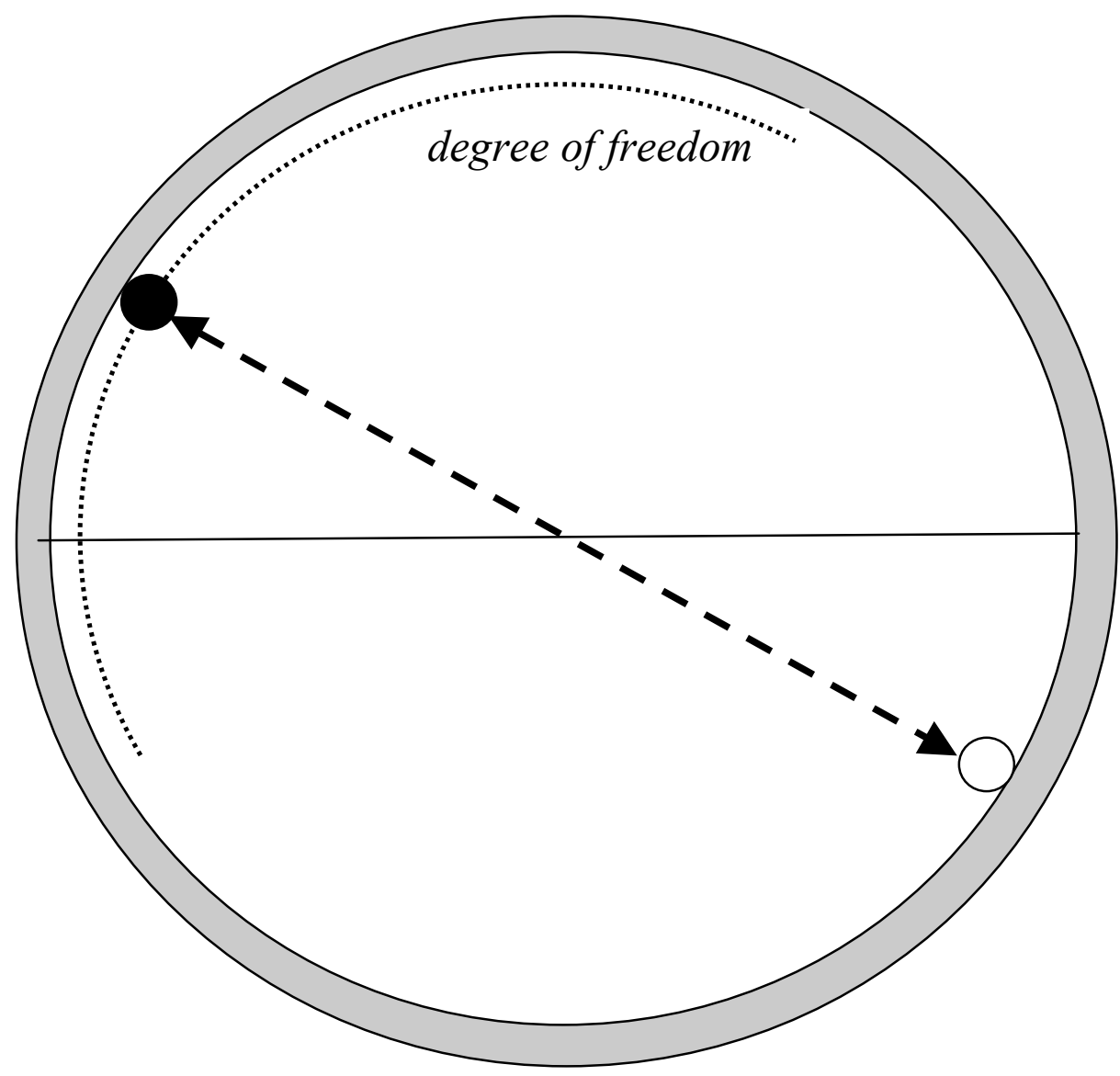

Fig. 2: a system consisting of two mutually repelling billiard balls on a round table. The system has one degree of freedom: the position of one of the balls along the perimeter (the other ball is always on the opposite side). Observing one ball to be in the upper or left half of the circle "collapses" the state of the second ball to the lower or right half.

This example does not yet illustrate non-locality, as the balls remain in local contact. Let us then imagine two billiard balls that repel each other, e.g. because they have the same electrostatic charge. Let us assume for simplicity that the billiard table has a round, circular shape. When the two balls are dropped at random positions on the table, the repulsive force will make them move away as far as possible from each other, until they reach the circular border of the table, where they will remain at opposite ends (Fig. 2). The balls can still move along the circle, but always together so that the distance between them remains maximal. That means that they are "entangled" in such a way that together they have only a single degree of freedom left: their position along the circle. An observer who would examine the upper half $(\uparrow)$ of the circle, and find ball $x$ there would be able to deduce that another observer examining the lower half $(\downarrow)$ at exactly the same instant would find the ball $y$ there. Vice versa, if the first observer would find $y$ in the upper section, it would follow that the second observer would find $x$ in the lower section.

We could describe this situation as a superposition of the eigenstates of this observation:

$$
|x \uparrow>| y \downarrow>+|x \downarrow>| y \uparrow>
$$


The discovery of $x$ in the top section $\uparrow$ would collapse this superposition to the first part of the above sum, thus also collapsing the state of $y$ to being present in the lower half. Note that if instead we had measured the presence of ball $x$ in the left half of the circle $\mid x \leftarrow>$, then we would be certain that ball $y$ would be in the right half $\mid y \rightarrow>$. The reason is that the constraint upon the two-component system merely says that the one component must be opposite to the other, so that their centre of mass always remains in the middle of the circle. This is similar to the classic example of quantum entanglement (Wiseman, 2006), where the constraint is that the total spin of the system consisting of $x$ and $y$ must be zero, meaning that spin up for $x$ implies spin down for $y$, while spin left for $x$ implies spin right for $y$. Such a global constraint creates a coherence or correlation between the parts of the system.

Since $x$ and $y$ are spatially separated, the correlation between their states is non-local. Of course, the correlation was initially created by a local interaction (between electrostatic forces). Yet, the same applies to non-local correlations in quantum systems, where the components first need to interact locally before their states can become entangled (e.g. particles with opposite spins are created from the decay of a single spin zero system).

This example may still appear too simple, given that an observer could in principle observe the positions of both balls at the same time. Let us then investigate a more complex system, where macroscopically observable properties emerge from non-observable, microscopic interactions.

\section{Rayleigh-Bénard convection}

Rayleigh-Bénard convection (Bodenschatz, Pesch, \& Ahlers, 2000; Nicolis \& Prigogine, 1977) is a classic example of self-organization in which a complex system reaches a bifurcation point, after which it settles in a new, coherent regime in which its initially independent components have become non-locally correlated. The phenomenon appears in a liquid that is heated evenly from below while it cools down evenly at its surface. The heat added to the bottom layer of the liquid must be transported to the top layer in order to be dissipated at the cool surface. Initially, this heat transport can take place by conduction, in which the molecules in the warmer liquid at the bottom pass on their kinetic energy to adjacent molecules until it reaches the surface. But as the heating is increased, the gradient or temperature difference $T$ between bottom and surface eventually becomes too large, and conduction is no longer sufficient. Transport of the heat can now only happen by convection: warmer (and thus lighter) liquid from the bottom floating up to the surface where it can release its heat to the cooler air above, while cooler (and thus heavier) liquid sinks down to the warmer bottom, where it collects heat.

These opposite movements at first sight create a conflict: the cool liquid moving down obstructs the warm liquid moving up. The "solution" to the conflict is that the movements of liquid coordinate so as to produce a circular flow: warm liquid flows up in one spot, cools down at the surface, moves sideways to a different spot where it now joins a cool, downward flow that reaches the bottom; there it warms up again, moves sideways in the opposite direction, back to the spot 
where it joins the initial upward flow, and so on (Fig. 3). This produces a "roll" of liquid circulating between surface and bottom. If the container is sufficiently large, the liquid will self-organize into a series of such parallel rolls, or, depending on the shape of the container, hexagonal cells. For simplicity, we will here examine the case of the rolls, so that we can depict the whole system in two dimensions: vertical, representing the temperature gradient, and horizontal, representing the points where rolls start and end. A roll rotating in a clockwise manner will normally be adjacent to a roll that rotates counterclockwise, and vice versa. Thus, the liquid spontaneously subdivides into a series of coordinated, parallel rolls

First, we can note that the flows in the different regions of the container are correlated. If we know the width of a roll (which will depend on the properties of the liquid and the container), then it suffices to know the speed and direction of flow in one region to derive the speed and the flow in any other region. Initially, a single roll may form somewhere in the liquid, but as its upwards and downward flows rub against the adjacent liquid, this part of the system too will start to move in the same direction, creating a parallel roll, which in turn will pass on its direction of movement to its neighboring region, until the whole container is filled with coordinated rolls. Thus, the correlation between neighboring regions propagates until it covers the whole system. This feature of selforganization in which a local order spreads can be expressed by the correlation length: the average distance over which parts of the system are still correlated. As self-organization propagates further, correlation length increases until it reaches the full size of the system. At that moment, the order is no longer local, but global or distributed. Using quantum terminology, we might say that the correlation has become non-local: if we know the direction of flow in one region, then we can instantly deduce the direction of flow in any other region, no matter how far apart these regions are.

A second crucial feature is that the direction of flow is not determined by the initial state of the system. The temperature difference $T$ plays the role of an order parameter: as its value increases, the system moves from an equilibrium state $s_{0}$ without convection flows to a bifurcation (Fig. 1), with two possible outcomes: a clockwise flow $s_{1}$ and a counterclockwise flow $s_{2}$. The state $s_{0}$ without flow becomes unstable after the bifurcation point. Therefore, the system will have to "choose" one of the two options $s_{1}$ or $s_{2}$. This is normally caused by an unobservably small, microscopic fluctuation, which makes that perhaps a few more liquid molecules move up rather than down in one particular spot. Because of the instability caused by the temperature gradient, this fluctuation is magnified into a macroscopic flow, driven by the heat energy that is continuously added to the liquid from below and that maintains the system far from equilibrium. As a result, the symmetry between clockwise and counterclockwise - or up and down - is broken: the system has opted for the one and rejected the other.

Let us represent this using a simplified quantum formalism. Consider a single convection roll, which we divide into a left-hand side $(x)$ and a right-hand side $(y)$. If the liquid is in the clockwise regime $s_{1}$, then it moves upward on the left of the roll: $\mid x \uparrow>$, and downward on the right: $\mid y \downarrow>$ (Fig. 3):

$$
s_{1}=|x \uparrow>| y \downarrow>
$$




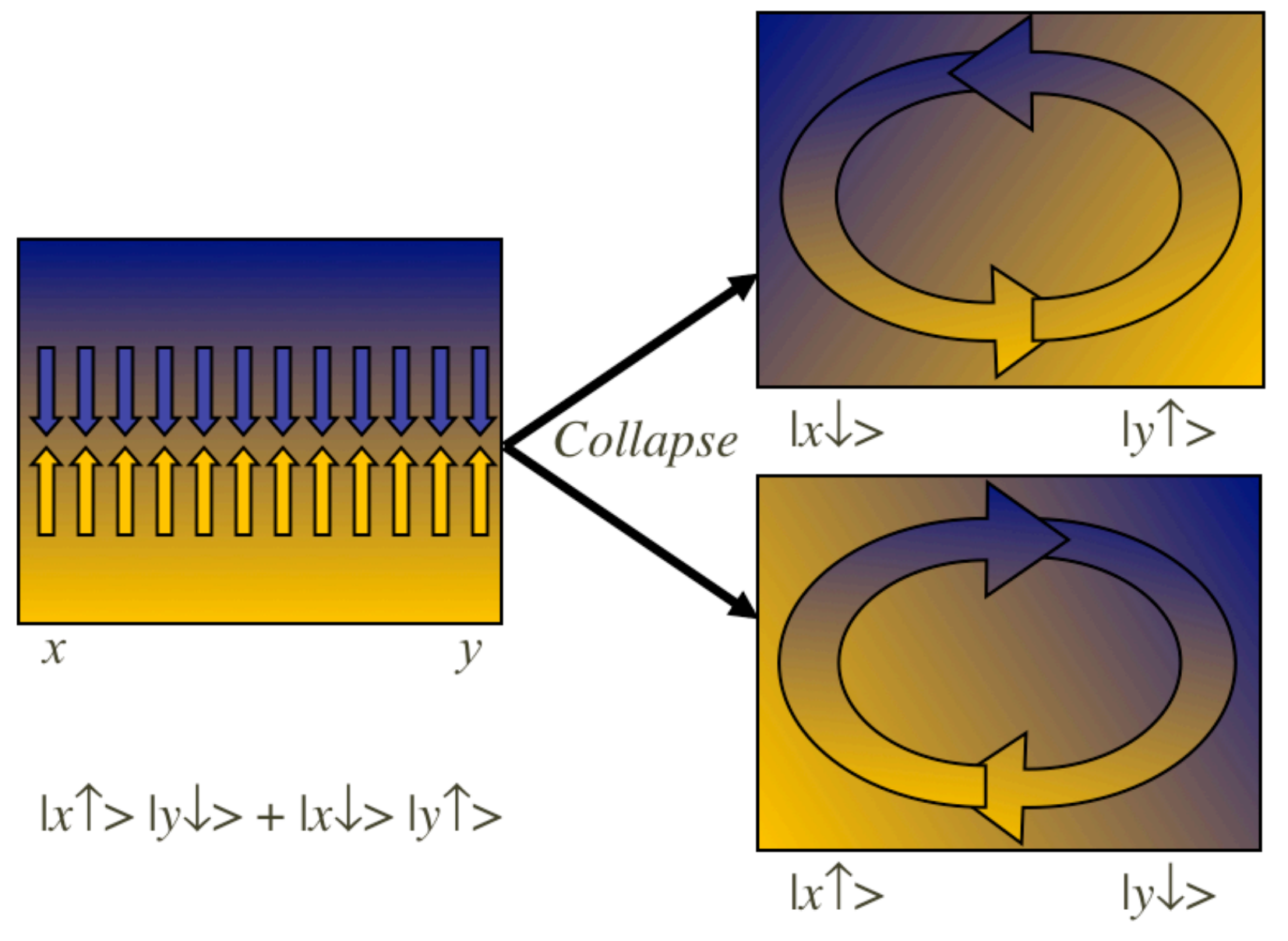

Fig. 3: The Rayleigh-Bénard instability. As the temperature difference between bottom (light, yellow) and top (dark, blue) of a liquid increases, heat must be transported by a convection flow (arrows). There are two possible solutions to this problem, a clockwise flow (bottom-right picture) and a counterclockwise flow (topright). In the first, cold liquid moves down on the left side (x) of the container, and up on the right (y) side: $\mid x$ $\uparrow>\mid y \downarrow>$, and vice-versa for the second. The original, unstable configuration can be seen as a superposition of both states that must collapse to one of them.

Vice versa for the counterclockwise regime:

$$
s_{2}=|x \downarrow>| y \uparrow>
$$

Let us go back to the unstable regime $s_{0}$ where the convection flow has not yet started and the symmetry between left and right has not yet been broken. We argued earlier that this "undecided" state on the border between two basins of attraction could be seen as a superposition of the two attractor states at the center of these basins:

$$
s_{0}=s_{1}+s_{2}=|x \uparrow>| y \downarrow>+|x \downarrow>| y \uparrow>
$$

This superposition describes an entanglement between the two regions $x$ (left) and $y$ (right). When the value of the order parameter $T$ is increased, this state will collapse to either $s_{1}$ or $s_{2}$. That means that 
the two separate regions $x$ and $y$ simultaneously opt for a certain flow direction, but such that the one is always the opposite of the other.

Note that this is similar to the classic Einstein-Podolsky-Rosen situation in which the spin of two entangled particles $x$ and $y$ is measured (Wiseman, 2006). Because their total spin is 0 , any measurement establishing that the one has spin up $\mid x \uparrow>$ instantaneously collapses the other one to its spin-down state $\mid y \downarrow>$. However, what creates the true "paradox" in this EPR set-up is that the observer could as well have measured a different property, such as the spin in the left-right direction. Here too a result of "left" for the one would have implied a collapse to "right" for the other, and vice versa.

Complementary properties are properties that cannot be observed simultaneously. For example, the set-up necessary to measure the wavelike properties of an electron (such as its momentum $p$ ) is incompatible with the set-up necessary to measure its particle-like properties (such as its position $x$ ). Therefore, the wave and particle aspects of quantum systems are said to be complementary: whenever we see the one, we cannot see the other, yet both are necessary to fully understand the behavior of the system. A precise observation result for one of a pair of complementary properties (like position and momentum, or spin in the vertical and in the horizontal directions) implies that the result for the other one becomes completely indeterminate. This is a more general formulation of the famous Heisenberg uncertainty principle (where $\Delta x$ represents the uncertainty in the measurement of position $x$ ):

$$
\Delta x . \Delta p \geq h / 4 \pi
$$

Can we find an equivalent of complementary properties that describe the emergence of a convection roll? Up to now, we distinguished the flow directions: clockwise $s_{1}$ and counterclockwise $s_{2}$. But the system is also characterized by the temperature difference between the lower layer of the liquid and the higher layer. This difference is driven by the parameter $T$ (temperature difference between heated bottom and cool surface). However, it also depends on the state of system. In the state $s_{0}$ without convection, there is a large temperature difference, because heat cannot travel easily from bottom to top. In the states $s_{1}$ and $s_{2}$, on the other hand, there is a small temperature difference, because warm liquid from the bottom is continuously transported to the cool surface, and vice versa (Fig. 3). The greater the heating and thus $T$, the faster the warm water will move to the surface to mix with the cooler water there. Thus, in the convection states there is a constant mixing of temperature layers that compensates for any increases in $T$. If an observation consists in registering the temperature in the lower and higher layers of the liquid, then we might say that the size of $T$ is not observable in the convection state, while its value is clearly determined in the equilibrium state $s_{0}$. Let us for simplicity distinguish two "eigenstates" of the observable $T$ : $\mid T$-large $>$ and $\mid T$-small $>$ (where "small" $T$ is still large enough to sustain convection). In that case, the convection state $s_{1}$, which is an eigenstate for the observable "direction of flow", becomes a superposition state for the observable $T$ :

$$
s_{1}=\mid T \text {-large }>+\mid T \text {-small }>
$$


Bringing back the convection state to the equilibrium state $s_{0}$ would in a sense "collapse" the superposition, because in this state we find either $\mid T$-large $>$ or $\mid T$-small $>$. This might be achieved as follows. We could temporarily interrupt the convection flow by inserting parallel horizontal plates that stop the liquid from moving in the vertical direction. After the plates have been inserted, we can measure the temperature difference between upper and lower layer, and determine whether the result of this observation is $\mid T$-large $>$ or $\mid T$-small $>$. But in this state we can no longer determine the result of the complementary observation $\mid x \uparrow>$ or $|x \downarrow\rangle$, because there is no flow. After the removal of the plates, the state will collapse again to one of the two flow states $s_{1}$ or $s_{2}$, but the result will be uncertain. Thus, the two observations of flow direction and temperature gradient are complementary, and a determinate result for the one implies an indeterminate one for the other. It would be an interesting exercise to check whether such a system could be made to violate the Bell inequalities that are the classic way to characterize quantum non-locality (Wiseman, 2006), just like the Bellviolating macroscopic system proposed by Aerts (1982).

\section{Other examples of complex phenomena}

There are many similar examples of self-organization in complex systems that exhibit quantum-like properties. A straightforward analogue to Bénard convection can be found in the lanes that spontaneously form in pedestrian traffic (Helbing, 2001; Helbing \& Molnar, 1998). Assume that a crowd of people needs to pass through a relatively narrow space, such as a street or square, but that half of the people move in one direction (say north to south) and half in the opposite direction (south to north). Initially, we are in a state of friction or mutual obstruction, as people will constantly need to change course in order not to bump into others. However, after a while their movements become more coordinated, as people moving in a given direction will start to follow each other, while staying out of the way of the people moving in the opposite direction. Thus, the available space selforganizes into two or more parallel "lanes", so that people in one lane all move in one direction, while those in the adjacent lane move in the opposite direction. This is similar to the liquid splitting up in parallel upward and downward flows, albeit it with the difference that individuals reaching the end of a lane/flow do not change direction and come back via the adjacent lane. Still, the initial separation between the lanes exhibits a similar kind of bifurcation dynamics, given that the same lane could have been used for either north-south or south-north traffic.

Initially, both options are equally likely, and it is some unpredictable fluctuation that will break the symmetry, making that in a given lane a few more people move in one direction than the other. As people tend to follow others that move in the same direction in order to avoid collisions, this chance fluctuation will be amplified until it dominates the length of the lane. Again, we could say that the initially uncoordinated movement corresponds to an indeterminate superposition state, which then "collapses" to one of several possible eigenstates characterized by a determinate flow for a given lane. The order parameter that forces the collapse in this case is the density of pedestrian traffic: the more people try to cross a given area, the more friction will be caused by people bumping 
into each other, and therefore the more people will tend to follow others moving in the same direction.

Such self-organization through the non-linear amplification of microscopic fluctuations is what Prigogine called "order through fluctuations" (Nicolis \& Prigogine, 1977). The symmetry breaking that it forces can be understood through what is called a "winner-takes-all" dynamics. This describes a situation in which several possible configurations are competing to take over a given assembly of components (such as molecules, drops of liquid, or people). The amplification is typically caused by a positive feedback or chain reaction in which the number of new "recruits" to the spreading configuration is proportional to the number that is already there, so that its growth is exponential. Components are typically recruited the moment a majority of their neighbors has been recruited. The first configuration to start growing will recruit most components, and thus extend the neighborhood in which it can make more recruits. Thus, it will encroach upon any configuration that started later and that therefore was able to recruit only a smaller number of neighbors. As a result, the first configuration (or the one that for some other reason managed to grow more quickly) will eventually overtake and erase any rival configurations. Thus, there will be a single "winner" that takes over all the components.

A classic example of this type of dynamics is opinion formation in a social group. Suppose that initially people have different opinions, but that they are inclined to conform to the opinion of their neighbors. If by chance, a small group of neighbors have the same opinion, their influence on their neighbors will be larger than the one of other neighbors that all have different opinions. Thus, the group will grow. Because of the positive feedback dynamics sketched above, eventually they will overtake the whole group so that everyone will settle on the same opinion, a process called "conformist transmission" (Henrich \& Boyd, 1998). Similar dynamics can be seen in the Ising model for magnetization, in which spins tend to align to the direction of spin (up or down) of their neighbors. Depending on how sensitive spins are to random fluctuations and to the influence of their neighbors, this may lead either to irregular zones in which molecules locally have the same spin, which is different from the one in other zones, or to a completely homogeneous outcome in which spins are globally aligned. In all these cases, we start from an indeterminate state where spins or opinions are randomly distributed, which then "collapses" to a single direction or opinion, thus breaking the symmetry of the initially homogeneous distribution, while creating a global correlation between all the regions.

A similar dynamics seems to occur in the brain. According to the global workspace theory, which is increasingly supported by empirical evidence (Dehaene, 2014; Sergent \& Dehaene, 2009), we become conscious of some perception or thought when it manages to win the competition for attention from rival stimuli. It thus comes to dominate the "global workspace", which is a kind of central neuronal crossroads from which commands are broadcasted to other parts of the brain. Many subconscious processes go on in parallel, but from those that reach the global workspace only the "winner-taking-all" can dominate consciousness. That is why consciousness is sequential: we can only be fully conscious of a single thought at a time, even while the subconscious activity in our neural networks outside the workspace is massively parallel. 
This is illustrated by a classic example that has clear similarities to both quantum processes and self-organization: Gestalt perception (Kruse \& Stadler, 2012; Stadler \& Kruse, 1990). Our consciousness does not see images as detailed arrays of pixels, parts or nuances, but as coherent wholes: clear figures with an unambiguous interpretation that are called "Gestalts". When an image is ambiguous, in the sense that it can be interpreted in more than one way, we are normally aware of only a single interpretation at a time. Well-known examples of such ambiguous figures are a shape that looks like either a rabbit or a duck, or one that resembles either a vase or two people's profiles facing each other. While we are able to perceive both Gestalts, we cannot do so simultaneously. Only one Gestalt can win the competition for global workspace domination.

Such an ambiguous figure can be represented as a superposition state, e.g. |rabbit $>+\mid$ duck $>$. But consciousness functions like a quantum observation that can only perceive eigenstates: |rabbit> or $\mid$ duck $>$. The process of reaching a conscious interpretation "collapses" the superposition state to one of the eigenstates, and this in an intrinsically unpredictable manner.

The implied symmetry breaking is perhaps illustrated most clearly by another ambiguous figure, the Necker cube (Fig. 4). This is a two-dimensional geometrical figure consisting of two connected squares, which the brain tends to interpret as a view on a three-dimensional cube. However, there are two ways of "seeing" the cube, one in which the left square appears as the front side of the cube (implying that the right square represents the back side), and one in which the right square appears as the front. The two interpretations are perfectly symmetrical, and there is no reason why the one would be preferred to the other. Yet, the winner-takes-all dynamics implies that only one can be present in consciousness at a given time. Thus, the initial symmetry must be broken, in a way that is intrinsically unpredictable.

This example also illustrates non-locality. Suppose we decompose the figure into left and right squares. Because of their connecting lines, the squares are "entangled". The initial state of our perception, before it reaches consciousness, is as yet indeterminate as to which square will appear as "front" and which as "back". Thus, we might describe it as a superposition state:

$$
\mid \text { left-front }>\mid \text { right-back }>+\mid \text { left-back }>\mid \text { right-front }>
$$

The operation of reaching consciousness collapses this state to one of the two eigenstates. Thus, the perception of the left square as being "front" forces the state of the right square to instantaneously collapse to "back". But left and right are separated by a finite distance, so this process is in a sense "non-local". 

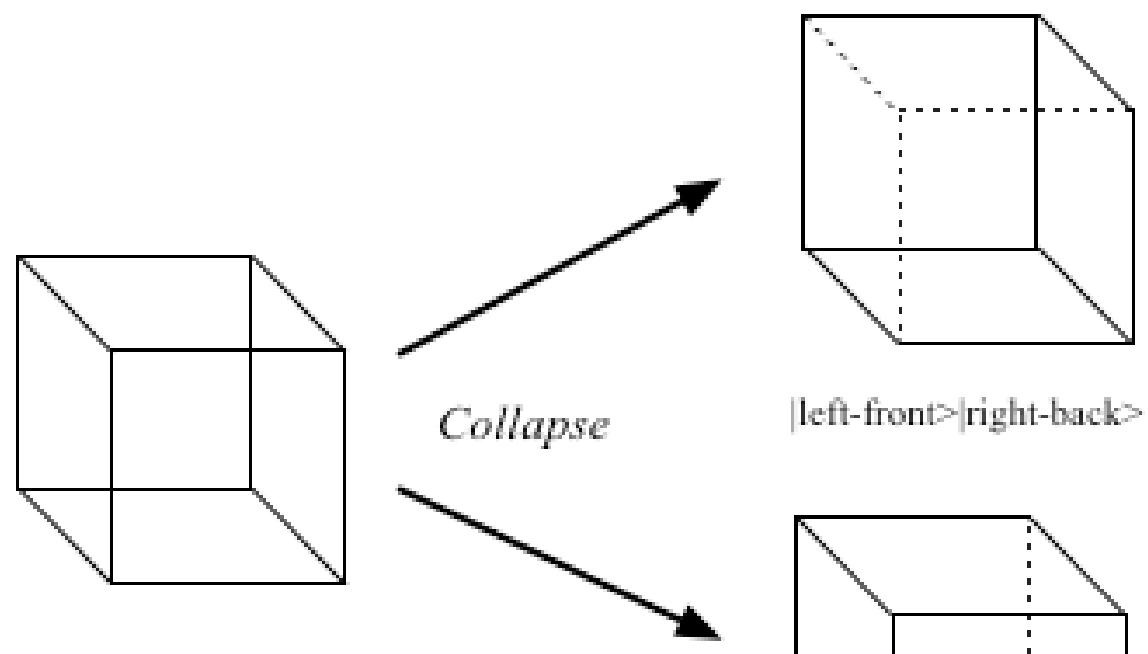

|left-front $>$ right-back $>$

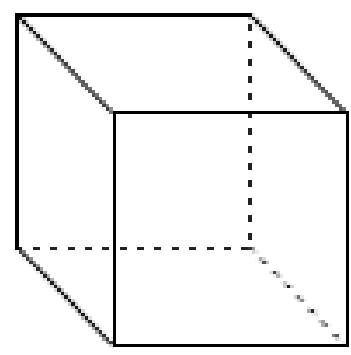

|left-back $>$ right-front>

Fig. 4: The Necker cube perceptual instability. The figure with the two connected, parallel squares on the left is interpreted by the brain as a view on a cube. However, there are two ways to "collapse" the ambiguous figure: either into a cube where the left square represents the front of the cube (top-right picture), and the right square the back, or a cube where front and back are switched (bottom-right picture).

\section{Some reflections on quantum field theory}

The above examples seem to make a good case for a parallelism between complex processes of selforganization and quantum observation processes. The question is whether this correspondence points to a fundamentally shared dynamics. Let us therefore examine the differences between the two domains, and see whether these can be eliminated. A first difference, the instantaneity of collapse in the quantum realm vs. an extended process of self-organization, is perhaps less fundamental than it appears. We noted earlier that a quantum observation is only instantaneous as a mathematical operation, but that its physical realization always has a finite duration. We also noted that at no point is information transmitted from one part of the system to another at a speed faster than light. The same applies to self-organization, where correlations between distant regions may emerge very fast, because of non-linear amplification, but the propagation of order or alignment still uses local interactions and therefore needs a finite time to reach all regions. Yet, once the correlation is global, we can forget about the finite process that produced it, and immediately deduce the state of one component from our observation of another one.

Another difference between the two types of processes is that the one is induced by an observation, while the other happens spontaneously - albeit typically driven by the change in value of an order parameter affecting the system. An observation implies an interaction between the 
quantum system being measured and some apparatus or set-up that necessarily affects the system being measured. In that sense, an observation can be seen as an external dynamics imposed by some observer on the system that forces the system to "collapse" and thus make a choice between the eigenstates that make up its initial superposition state. This does not look fundamentally different from the dynamics externally induced by the change of an order parameter that forces it to choose between attractors. This point is confirmed by the more recent interpretation of wave function collapse as a process of "decoherence" (Joos et al., 2013; Zurek, 2003), in which the noisy interactions between the quantum system and its environment gradually erase the coherence that existed between the different parts of the superposition state or wave function.

This brings us to a fundamental question: what causes the system to choose for one option rather than another? If we assume perfect symmetry between the options, in the sense that none of the solutions is in any way preferable to the others, then there does not seem to be a cause. Such spontaneous symmetry breaking (Castellani, 2003) appears like a fundamentally indeterministic, uncaused process, or what I have previously called a "distinction creation" (Heylighen, 1989, 1990; Heylighen et al., 2007). In self-organization, the cause is normally assumed to be some microscopic, unobservable fluctuation (Nicolis \& Prigogine, 1977), similar to the random distribution of molecular movements that cause Brownian motion in a liquid. The equivalent in quantum mechanics would be a hidden variable, a microscopic property that we cannot observe but that affects the outcome of the observation. However, the Bell theorem has established that if hidden variables are responsible for the paradoxes of entanglement, then these hidden variables must be non-local (Wiseman, 2006), which would contradict some of the deepest principles underlying physical theories. Therefore, hidden variables are generally rejected as an explanation for quantum indeterminacy.

Aerts has proposed an elegant hypothesis to resolve the problem: the hidden measurement approach (Aerts, 1998). This assumes that the unobservable properties that determine the outcome of an observation are not hiding inside the quantum system, but inside the measuring apparatus. This makes sense, given that a microscopic quantum system, such as an electron, hardly offers any "room" to hide anything, while an observation apparatus constitutes a complex, macroscopic system of which we can never determine the full microstate. Since the outcome of the measurement depends on both system and apparatus, it seems reasonable to assume that different, albeit nondistinguishable, microstates for the same apparatus would result in different outcomes.

To make things more concrete, Aerts proposes a toy model that illustrates how the hidden measurement approach might give rise to some of the probability distributions predicted by quantum theory. In this model, the (deterministic) state of the quantum system is somehow registered on a particular spot along an elastic string that is extended inside the apparatus. (This can be visualized with the set-up in Fig. 2, where we imagine that one of the balls, representing the system, would "fall" onto the horizontal line that represents the "string"). But the apparatus can only register two possible outcomes (eigenstates), "spin-left" or "spin-right". This happens when the string breaks in two. If the spot is found on the left piece of string, the registered outcome is "spin-left", and vice versa. The indeterminacy is introduced by the fact that we do not know where along its length the string will break. 
This model actually provides a beautifully simple illustration of our initial problem of symmetry breaking. When the tension on a string is increased, there will inevitably come a moment when the string will break, thus creating a distinction or differentiation. Let us assume that the string is homogeneous: every segment is as strong as any other segment. Therefore, there are no a priori "weak spots" that would break under a lower tension than the other regions. Because the string is elastic, no matter where the tension is exerted, this tension will spread evenly over all segments. Indeed, imagine that the tension would be higher in segment $A$, so that $A$ would be extended more. That means that $A$ will pull harder on its neighboring segment $B$ which is as yet less extended, and thus has more elastic capacity to absorb the tension, until it is equally tense as $A$. Thus, any local inhomogeneity would immediately be globally diffused, so that the tension remains homogeneous. But that means that any segment of the string is just as likely (or unlikely) to break as any other. Yet, as the tension is increased, the string has to break in a particular spot, which therefore is selected out of a continuum of equivalent spots. This is similar to the symmetry breaking during a bifurcation, with the increasing tension playing the role of the order parameter forcing the system to make a choice. The only difference is that in this case the number of possible choices is continuously infinite, rather than finite.

In an actual macroscopic experiment, we would explain the symmetry breaking by some microscopic inhomogeneity in the rubber of the string, or perhaps a fluctuation in the distribution of molecular forces in the string. In quantum mechanics, we attribute it to some peculiarity of the observation process, perhaps explainable by the way the apparatus perturbs the system. But there is a deeper theory suggesting that the symmetry breaking is truly spontaneous, and does not need any external factors: (relativistic) quantum field theory (Mandl \& Shaw, 2010). The mathematics of this theory is so complex that no one really seems to have dared to propose a systematic or intuitive interpretation of its results. Yet, these results are so amazingly wide-ranging and empirically accurate that it is probably the most reliable theory in the whole of science. But we do not need to understand the mathematical derivation to appreciate some of the phenomena it explains.

Perhaps the best-known case is radioactive decay: a radioactive atom is by definition unstable, which means that it may disintegrate into smaller particles at any moment in time. However, it is impossible to determine at which time such decay will happen. We only know that there is a fixed probability for an atom to decay during a given time interval. The half-life of an isotope denotes the interval for which that probability is exactly $50 \%$. That means that after that period, about half of the atoms in a sample will have decayed. Yet, these atoms were absolutely identical as far as the forces responsible for radioactivity are concerned. The initial homogeneity or symmetry between the atoms has been broken: some have decayed, others have survived. But here we cannot find any internal or external cause responsible for this difference: no observation, no order parameter, no microscopic inhomogeneity, no perturbation... has affected the atoms. Each atom on its own, independently of outside influences, has decided at some random moment to either fall apart or remain.

A less well-known, but actually more common, example of such an intrinsically indeterministic process is the "quantum jump" through which an excited atom or molecule falls back to a lower level of energy. Any energy level higher than the ground state is unstable, and will 
eventually fall back to its ground level while emitting one or more photons to carry away the excess energy. However, the time of this decay and the direction in which the photon is emitted are essentially unpredictable. Ironically, although the emission of such "quanta" of energy is what gave quantum mechanics its name, the process cannot be explained within conventional quantum mechanics. Indeed, the evolution of a quantum state as described by the Schrödinger equation is intrinsically continuous, while discontinuities only appear when an observation collapses the state to an eigenstate. But the discontinuous emission of a photon by an excited state happens spontaneously, without any observation taking place. It therefore has to be explained by quantum field theory.

The only "cause" for such symmetry breaking this theory proposes is a so-called quantum fluctuation of the vacuum. In field theory, a vacuum is the zero-energy state of the field. Any positive energy injected into the field creates an "excitation", which classically takes the form of a wave, but quantum mechanically can be interpreted as a particle (e.g. a photon). Now, the Heisenberg uncertainty principle for the complementary properties of time and energy states that when time intervals become arbitrarily small, the uncertainty on the energy present in that time interval becomes arbitrarily large. That means that if we consider the vacuum across a sufficiently short interval, the energy present during that interval can take on values large enough to produce "virtual particles". These are excitations of the field that are so short-lived that we cannot directly observe them, because they decay almost as soon as they appeared. But during their brief life, they can interact with a system such as an atom in an excited state, and trigger the decay of that state. Thus, quantum field theory suggests that even emptiness or nothingness (i.e. absence of any energy, matter or other differentiating feature) can temporarily fluctuate by randomly producing virtual particles or waves that affect unstable systems, and that may force them to "collapse".

How does this relate to self-organization? A typical self-organizing system, such as a liquid or an array of spins, consists of many interacting components distributed across space. Through local interactions, a change in the state of a component tends to propagate to its neighboring components, thus spreading like a wave across the medium. Examples of such waves are the "phonons", which are particle-like vibrations propagating in some solid medium. This medium is discrete, consisting of separate components, such as molecules. A field is a continuous medium, but that can be seen as the limit of such a discrete medium when the distance between the components goes to zero. For example, it has been shown that the classic Ising model, which is used to illustrate the selforganization of magnetization via the propagation of magnetic alignment between neighboring spins, becomes isomorphic to a quantum electromagnetic field in such a limit. Thus, fields and complex dynamic systems appear like different ways to model a dynamics of fluctuation and propagation, where fluctuation plays the role of the initial event that precipitates the symmetry breaking, while propagation across a medium is the mechanism that amplifies this random change, and creates a new coherence or coordination between remote regions. A classic application of this in physics are phase transitions, such as the transition from (disordered) liquid to (coherent) solid, which can be modeled using both non-linear statistical mechanics and an equivalent of QFT (Zinn-Justin, 1996). 


\begin{tabular}{|l|l|}
\hline Quantum phenomena & Complex dynamics/self-organization \\
\hline Superposition state & Indeterminate state \\
\hline Uncertainty & Bifurcation \\
\hline Wave function collapse & Reaching of attractor \\
\hline Entanglement & Emergent constraint \\
\hline Non-locality & Non-local correlations \\
\hline Observation & Order parameter \\
\hline Symmetry breaking & Symmetry breaking \\
\hline Eigenstate & Attractor state \\
\hline
\end{tabular}

Table 1: some correspondences between quantum and self-organizing phenomena.

\section{Conclusion}

We have investigated the correspondence between fundamental aspects of quantum theory and related phenomena in the emerging theory of complex, self-organizing systems (Table 1). On the one hand, this helps us to better grasp the often counterintuitive behavior of quantum systems, because we now can see how they are analogous to macroscopic phenomena with which we have more direct experience. It also suggests new methods to perhaps analyze that behavior at a deeper level. Viceversa, the analogy with quantum mechanics suggest new ways of looking at complex phenomena, such as the self-organization of cognitive or social structures, thus extending the program that was initiated by quantum cognition (Aerts, 2009; Aerts, Gabora, Sozzo, \& Veloz, 2011; Bruza et al., 2015).

Quantum phenomena at the deepest level are characterized by the superposition principle. It implies that for any two states with determinate characteristics there exists a superposition state for which these same characteristics are indeterminate, meaning that an observation may produce either the one or the other result, in an unpredictable manner. This uncertainty is mirrored in complex systems by the phenomenon of bifurcation, which notes that under changes of an order parameter stable solutions may multiply, forcing the system to "choose" one of a number of a priori equivalent states. In both quantum and complex dynamics, the initially indeterminate state appears to "collapse" into one of the determinate outcomes, where it then remains. This means that the outcome is an attractor or eigenstate of the process that precipitated the collapse. The choice of one among several equivalent outcomes in both cases defines a symmetry breaking. In complex dynamics, this symmetry breaking appears to be initiated by a microscopic fluctuation, while in quantum mechanics it may be caused by the microscopic perturbation induced by the observation apparatus, or more fundamentally by the fluctuations of the vacuum implied by the Heisenberg uncertainty principle.

In self-organizing systems, the "collapse" into an attractor state produces a global or emergent constraint that induces a correlation between the components of the system. This is similar to the 
"entanglement" that can occur between the components of a quantum system, when the system as a whole is constrained to obey a global condition, such as having a total spin of 0 . Such correlations are non-local, in the sense that the states of spatially remote components depend on each other, and a determinate observation outcome (collapse) for the one forces a complementary outcome for the other, even when their individual states are initially indeterminate. It is not yet clear whether the correlations in self-organizing systems can exhibit the same paradoxical property expressed by the violation of the Bell inequalities that characterizes quantum non-locality (Aerts, 1982). This and the role of quantum fluctuations of the vacuum in triggering symmetry breaking appear like promising issues for further research.

\section{References}

Aerts, D. (1982). Example of a macroscopical classical situation that violates Bell inequalities. Lettere Al Nuovo Cimento (1971-1985), 34(4), 107-111.

Aerts, D. (1998). The hidden measurement formalism: what can be explained and where quantum paradoxes remain. International journal of theoretical physics, 37(1), 291-304.

Aerts, D. (2009). Quantum structure in cognition. Journal of Mathematical Psychology, 53(5), 314348.

Aerts, D., Gabora, L., Sozzo, S., \& Veloz, T. (2011). Quantum structure in cognition: fundamentals and applications. arXiv preprint arXiv:1104.3344.

Ashby, W. R. (1962). Principles of the self-organizing system. In H. von Foerster \& G. W. Zopf (Eds.), Principles of Self-Organization (pp. 255-278). Pergamon Press. Retrieved from http://csis.pace.edu/ marchese/CS396x/Computing/Ashby.pdf

Aspect, A. (2007). Quantum mechanics: to be or not to be local. Nature, 446(7138), 866.

Ball, P. (2012). Why Society is a Complex Matter: Meeting Twenty-first Century Challenges with a New Kind of Science (2012th ed.). New York: Springer.

Bedau, M. (2002). Downward causation and the autonomy of weak emergence. Principia, 6(1), 5. Retrieved from 
http://search.proquest.com/openview/e32baabe9f7b694db698fab5a4a17062/1?pq-

origsite $=$ gscholar $\&$ cbl $=2026966$

Bodenschatz, E., Pesch, W., \& Ahlers, G. (2000). Recent Developments in Rayleigh-Bénard Convection. Annual Review of Fluid Mechanics, 32(1), 709-778. doi:10.1146/annurev.fluid.32.1.709

Bruza, P. D., Wang, Z., \& Busemeyer, J. R. (2015). Quantum cognition: a new theoretical approach to psychology. Trends in cognitive sciences, 19(7), 383-393.

Castellani, E. (2003). On the meaning of symmetry breaking. Symmetries in physics: Philosophical reflections, 321-334.

Dehaene, S. (2014). Consciousness and the Brain: Deciphering How the Brain Codes Our Thoughts. Penguin.

Dirac, P. A. M. (1981). The principles of quantum mechanics. Oxford university press.

Eberhard, P. H., \& Ross, R. R. (1989). Quantum field theory cannot provide faster-than-light communication. Foundations of Physics Letters, 2(2), 127-149. doi:10.1007/BF00696109

Ghirardi, G. C., Grassi, R., Rimini, A., \& Weber, T. (1988). Experiments of the EPR Type Involving CP -Violation Do not Allow Faster-than-Light Communication between Distant Observers. EPL (Europhysics Letters), 6(2), 95. doi:10.1209/0295-5075/6/2/001

Haken, H. (1977). Synergetics: an introduction: nonequilibrium phase transitions and selforganization in physics, chemistry, and biology. Springer.

Helbing, D. (2001). Traffic and related self-driven many-particle systems. Reviews of modern physics, 73(4), 1067. Retrieved from http://rmp.aps.org/abstract/RMP/v73/i4/p1067_1

Helbing, D., \& Molnar, P. (1998). Self-organization phenomena in pedestrian crowds. arXiv preprint cond-mat/9806152. Retrieved from http://arxiv.org/abs/cond-mat/9806152

Henrich, J., \& Boyd, R. (1998). The Evolution of Conformist Transmission and the Emergence of Between-Group Differences. Evolution and Human Behavior, 19(4), 215-241. 
Heylighen, F. (1989). Causality as Distinction Conservation: a theory of predictability, reversibility and time order. Cybernetics and Systems, 20(5), 361-384. doi:10.1080/01969728908902213

Heylighen, F. (1990). Classical and nonclassical representations in physics II: quantum mechanics. Cybernetics and Systems, 21(5), 477-502. doi:10.1080/01969729008902255

Heylighen, F. (2001). The science of self-organization and adaptivity. The Encyclopedia of Life Support Systems, 5(3), 253-280. Retrieved from http://pespmc1.vub.ac.be/Papers/EOLSSSelf-Organiz.pdf

Heylighen, F. (2009). Complexity and Self-Organization. Encyclopedia of Library and Information Sciences, Third Edition (pp. 1215-1224). Taylor \& Francis. Retrieved from http://www.tandfonline.com/doi/abs/10.1081/E-ELIS3-120043869

Heylighen, F., Cilliers, P., \& Gershenson, C. (2007). Complexity and Philosophy. In J. Bogg \& R. Geyer (Eds.), Complexity, science and society (pp. 117-134). Oxford: Radcliffe Publishing,. Retrieved from http://arxiv.org/abs/cs/0604072

Hilborn, R. C. (2004). Sea gulls, butterflies, and grasshoppers: A brief history of the butterfly effect in nonlinear dynamics. American Journal of Physics, 72(4), 425-427. Retrieved from http://scitation.aip.org/content/aapt/journal/ajp/72/4/10.1119/1.1636492

Horodecki, R., Horodecki, P., Horodecki, M., \& Horodecki, K. (2009). Quantum entanglement. Reviews of modern physics, 81(2), 865.

Joos, E., Zeh, H. D., Kiefer, C., Giulini, D. J., Kupsch, J., \& Stamatescu, I.-O. (2013). Decoherence and the appearance of a classical world in quantum theory. Springer Science \& Business Media.

Kruse, P., \& Stadler, M. (2012). Ambiguity in Mind and Nature: Multistable Cognitive Phenomena. Springer Science \& Business Media.

Mandl, F., \& Shaw, G. (2010). Quantum Field Theory. John Wiley \& Sons.

Milnor, J. W. (2006). Attractor. Scholarpedia, 1(11), 1815. doi:10.4249/scholarpedia.1815 
Nicolis, G., \& Prigogine, I. (1977). Self-organization in nonequilibrium systems: From dissipative structures to order through fluctuations. Wiley, New York.

Sergent, C., \& Dehaene, S. (2009). PSYCHOLOGICAL SCIENCE Research Article Is Consciousness a Gradual Phenomenon? Evidence for an All-or-None Bifurcation During the Attentional Blink.

Stadler, M., \& Kruse, P. (1990). Theory of Gestalt and Self-organization. Self-Steering and Cognition in Complex Systems. Gordon and Breach, New York, 142-169.

Wiseman, H. M. (2006). From Einstein's theorem to Bell's theorem: a history of quantum nonlocality. Contemporary Physics, 47(2), 79-88.

Zinn-Justin, J. (1996). Quantum field theory and critical phenomena. Clarendon Press.

Zurek, W. H. (2003). Decoherence, einselection, and the quantum origins of the classical. Reviews of modern physics, 75(3), 715. 